\section{Fortschritte bei der Behand- lung des neugeborenen Fohlens mit respiratorischer Insuffizienz: Klinische Einschätzung}

\author{
P. C. Kosch ${ }^{1}$, Anne M. Koterba, T. J. Coons ${ }^{2}$ und A. I. Webb
}

Departments of Medical Sciences and Physiological Sciences ${ }^{1}$, College of Veterinary Medicine, and Respiratory Care Services, Shands

Teaching Hospital'2, University of Florida

\section{Einführung}

Die Erkrankungen beim neugeborenen Fohlen sind von beträchtlicher wirtschaftlicher Bedeutung, wobei sich die Mortalität laut Berichten zwischen 3,6 und 5,0 Prozent bewegt (Merkt und von Lepel, 1971; Platt, 1973; Rossdale und Leadon, 1975). Pathologische Zustände des Atmungsapparats treten mit großer Häufigkeit auf, und zwar als möglicherweise primärer Prozeß oder sekundär infolge einer anderen Gesundheitsstörung. Dubielzig (1977) gelangte zu dem Ergebnis, daß 34 von 36 Fohlen, die im Alter von weniger als fünf Tagen starben, sichtbare entzündliche oder nichtentzündliche Veränderungen der Lungen aufwiesen. Bei 35 von 38 septikämischen Fohlen, die weniger als eine Woche alt waren, stellte man bei der Autopsie pathologische Erscheinungen an den Lungen fest (Koterba, Brewer und Tarplee, 1984), wobei es sich in den meisten Fällen um eine schwere diffuse Pneumonie handelte. Auch chronische Alveolitis, Atelektase, Emphysem und Pleuritis wurden beobachtet.

Erkrankungen des Respirationstrakts kommen bei den Neugeborenen aller Spezies häufig vor. In der humanmedizinischen Neonatologie hat man die Sterblichkeit mit Hilfe der frühen Diagnose und der routinemäßigen positiven Druckbeatmung über einen Tubus und mechanische Ventilation von Säuglingen mit respiratorischer Insuffizienz in beachtlichem Maße senken können (Gregory, 1972). Die Fortschritte, welche die Veterinärmedizin bei der Anwendung dieser Lebensrettungsmaßnahmen bei neugeborenen Fohlen gemacht hat, sind nicht so groß. Die Errungenschaften der humanmedizinischen Neonatologie haben jedoch zusammen mit dem Wert einzelner Tiere die Forderung nach Maßnahmen bei der Intensivüberwachung des Neugeborenen auf einer höheren veterinärmedizinischen Entwicklungsstufe immer stärker werden lassen. Der Vorgang, Verfahren zur Beurteilung und Therapie auszuarbeiten, die - ausgehend von einem $1500 \mathrm{~g}$ schweren Säugling auch bei einem $45 \mathrm{~kg}$ schweren Fohlen in einem Stall angewandt werden können, nimmt jedoch viel Zeit in Anspruch.

\section{Zusammenfassung}

Es werden die Fortschritte in der Beurteilung von neugeborenen Fohlen mit respiratorischer Insuffizienz besprochen. Die Hauptursachen der Dyspnoe werden aufgeführt und im Hinblick auf die sich untereinander ähnlichen respiratorischen Symptome diskutiert, die Fohlen mit diesem klinischen Syndrom zeigen. Vorbericht, klinische Untersuchung, klinische Pathologie, Röntgenuntersuchung des Thorax und Blutgasanalyse werden als wichtige Kriterien bei der Bewertung des Zustandes solcher Fohlen besprochen. Die Fohlen mit Erkrankung des Atmungsapparates werden auf der Grundlage der klinischen Erscheinungen und der Blutgasanalysewerte in drei Hauptgruppen eingeteilt. Die Beurteilung dyspnoischer Fohlen wird in Einzelheiten dargelegt, um zu einer exakten, ätiologischen Diagnose zu gelangen und um die Entscheidung zu erleichtern, ob das Fohlen hinsichtlich der Atmung unterstützt werden muß.

Diese Abhandlung soll einige wichtige Gesichtspunkte der respiratorischen Insuffizienz beim Fohlen herausstellen und dient dazu, einige diagnostische Vorgehensweisen zu besprechen, die sich als zweckmäßig erwiesen haben. Webb, Coons, Koterba und Kosch (1984a) stellen die therapeutischen Verfahren umfassend dar. Die Empfehlungen beider Arbeiten haben die mehrjährige Erfahrung aus der Behandlung einer großen Anzahl kranker Fohlen am Veterinary Medical Teaching Hospital der Universität Florida zur Grundlage. Rossdale (1980), Rossdale und Ricketts (1980) und Martens (1982) betrachten in ihren Arbeiten eingehender die Pathophysiologie der Atmung.

\section{Definition der respiratorischen Insuffizienz}

Die Adaptation an die extrauterine Umgebung läßt schlagartig grundlegende Umwandlungen der Lungenstruktur und -funktion erforderlich werden. Vor der Geburt sind die Lungen flüssigkeitsgefüllt und werden nur von einem geringen Teil Blut des Herzschlagvolumens durchströmt, in den ersten paar Minuten nach der Geburt muß eine groBe Flüssigkeitsmenge absorbiert und durch Luft ersetzt werden. Der Brustkorb weitet sich, und der Blutfluß durch die Lungen nimmt wesentlich zu (Avery, Fletcher und Williams, 1981). Die Lunge muß in kürzester Zeit zu einem Organ mit wirkungsvollem Gasaustausch werden, und wenn der Ablauf dieser Veränderungen versagt oder sich verzögert, führt dies in der Regel zu den Erscheinungen der Atmungsinsuffizienz.

Rossdale (1979) definierte die respiratorische Insuffizienz als Lungendysfunktion, die sekundär zur mangelhaften Arterialisation des Blutes durch ein Mißverhältnis zwischen Ventilation und Perfusion und physiologischen oder anatomischen Shunts führt. Zum selben klinischen Syndrom kommt es möglicherweise aber auch bei einer Funktionsstörung außerhalb der Lungen (wie einer Verlegung der oberen Luftwege oder einer Erkrankung der Pleura).

\section{Ätiologie und Pathogenese}

Tabelle 1 stellt eine Liste der Hauptursachen der respiratorischen Insuffizienz beim neugeborenen Fohlen dar. Das Membransymptom der Früh- und Neugeborenen ist zwar 
Primär

(A) Lungenerkrankung

Pneumonie

Membranerkrankung der

Früh- und Neugeborenen
Sekundär

persistierender fötaler Kreislauf

akuter Blutverlust, Anämie

metabolische Azidose, Hypoglykämie
Aspirationssyndrome (Mekonium)

vorübergehende Tachypnoe
(B) Störung außerhalb der Lunge

Verschluß der oberen Luftwege

(Choanenatresie, kollabierte Trachea)

Pleuritis zerebrale Hämorrhagien und/oder Ödeme

(Pneumothorax, Zwerchfellshernie)

Tab. 1: Ursachen der respiratorischen Insufizienz beim neugeborenen Fohlen

in der Humanmedizin wohlbekannt, es ist jedoch ungewiß, ob es klinisch auch beim Fohlen auftritt. Laut Berichten ist die Surfactantauskleidung bei Fohlen, die nach weniger als 300 Tagen Trächtigkeit zur Welt kommen, unvollständig, manchmal sogar auch am Ende der physiologischen Graviditätsdauer (Pattle, Rossdale, Schock und Creasy, 1975; Rossdale, Pattle und Mabaffey, 1967).

Eine verbreitete Folgeerscheinung einer pränatalen Asphyxie ist der Übertritt von Mekonium in die Aminoflüssig keit, welches dann aspiriert wird, wenn das Fohlen nach Luft ringt, und so die Ursache einer Pneumonie beim Neugeborenen sein kann. Bakterielle, interstitielle und Bronchopneumonien kommen oft bei ausgetragenen und frühgeborenen Fohlen vor. Unter Umständen sind sie von Fällen einer Aspirationspneumonie schwer zu unterscheiden und flammen manchmal unter klinisch dramatischem Verlauf auf. Eine Infektion kann sich das Fohlen pränatal, in tra oder post partum zuziehen.

Gelegentlich ist nur eine permanent erhöhte Atemfrequenz festzustellen, und alle anderen Anzeichen einer Störung am Atmungsapparat fehlen. Sie kann das frühe Symptom einer Pneumonie sein, und man muß alles nur Durchführbare tun, um diese Möglichkeit differentialdiagnostisch auszuschließen. Als häufigste Ursache einer vorübergehenden Tachypnoe wird die langsame Absorption von Flüssigkeit in der Lunge betrachtet (Klaus, Fanaroff und Martin, 1979). Der Zustand normalisiert sich gewöhnlich innerhalb von einigen Tagen. Die Autoren beobachteten idiopathisches Fieber und Tachypnoe bei zwei neugeborenen "Clydesdale-Fohlen" von derselben Farm. Die Erscheinungen verschwanden spontan nach zwei Wochen, und die einzige Behandlungsmaßnahme bestand darin, das Fell zu scheren, um die Körpertemperatur zu regulieren. Ausgedehnte diagnostische Untersuchungen erbrachten weder eine die Lungen betreffende noch anhidrotische oder infektiöse Primärerkrankung, und man nahm an, daß eine vorübergehende Störung der Thermoregulation dafür verantwortlich war. Die weitestverbreiteten Gründe einer Obstruktion der oberen Luftwege kurz nach der Geburt stellen kongenitale Mißbildungen wie Choanenatresie (Crouch und Morgan, 1983), Stenosen in den Nüstern und das Kollabieren der Trachea dar.

Viele schon vor der Geburt bestehende, nicht von den Lungen ausgehende Faktoren bewirken eine anormale Atemfrequenz oder einen unphysiologischen Atmungstyp. Zum
Beispiel regt die metabolische Azidose eine Hyperventilation an, eine Hypoglykämie kann Apnoe verursachen, und schwere kongenitale Herzanomalien können zu Lungenödem, Zyanose und erschwerter Atmung führen. Asphyktische Fohlen, an denen man erfolgreich Wiederbelebungsmaßnahmen durchgeführt hat, oder solche, bei welchen Anzeichen eines neonatalen Maladaptationssyndroms bestehen, ohne daß etwas von einer Asphyxieperiode während der Geburt bekannt ist, zeigen oft Tachypnoe, Apnoephasen und andere Erscheinungen von respiratorischer Insuffizienz. Einige dieser Atmungsanomalien treten wahrscheinlich sekundär infolge hypoxischer Schädigung der Neuronen in den Atemzentren auf. Als weitere Manifestation der Geburtsasphyxie können pathologische Lungenveränderungen, insbesondere die in ihrer Art nichtentzündliche fleckige Atelektase, angesehen werden.

\section{Klinische Beurteilung}

\section{Anamnese}

Gewöhnlich benötigt man einen genauen Vorbericht, um die Ursache der Atmungsinsuffizienz beim neugeborenen Fohlen herauszufinden. Ist die exakte Trächtigkeitsdauer bekannt, so hilft dies dabei, frühgeborene Fohlen von solchen differentialdiagnostisch $\mathrm{zu}$ trennen, die angesichts normaler Trächtigkeitsdauer relativ zu klein sind.

Pränatale Gesundheitsprobleme der Mutter wie Fieber, Scheidenausfluß und systemische Erkrankungen können Streßsituationen und/oder Infektionen beim Fötus Vorschub leisten. Geburtsstörungen, vorzeitige Lösung der Plazenta, Kompression der Nabelschnur und andere Geburtskomplikationen haben eventuell einen gewissen Grad von Asphyxie und hypoxischer Gewebeschädigung zur Folge. Verfärbte Amnion- oder Allantoisflüssigkeit deutet an, daß die Gefahr der Mekoniumaspiration besteht, und Anzeichen von Chorioamnionitis, plaqueförmige Veränderungen, unphysiologische Verdickungen oder Ödeme der Plazenta sollten als Hinweis für neonatale pathophysiologische Vorgänge betrachtet werden.

Man sollte auch den vorausgegangenen Verlauf der Erkrankung des Fohlens erfragen, ebenso bereits durchgeführte Behandlungen und Eigenarten des Betriebs. Nach Erfahrung des Autors waren mild verlaufende Infektionen der oberen Atemwege bei der Stute manchmal mit plötzlich aufflammenden tödlich verlaufenden interstitiellen Pneu- 
monien ihrer neugeborenen Fohlen verknüpft. Die Ätiologie ist ungeklärt, man vermutete jedoch den Grund in einer Virusinfektion.

Daß dem Fohlen Kolostrum fehlt, ist am von den Zitzen abtropfenden Kolostrum zu erkennen. Fohlen, die nur langsam zu saugen beginnen, nehmen in der Regel auch nicht ausreichend Kolostralmilch auf. Bei Fohlen mit Immundepression besteht ein erhöhtes Risiko bakterieller Infektionen, die sich oft in der Lunge manifestieren.

\section{Klinische Untersuchung}

Die ersten Untersuchungsschritte am Fohlen sollten rasch Klarheit darüber verschaffen, ob sofort Wiederbelebungsmaßnahmen erforderlich sind (Webb et al., 1984a). Atemfrequenz, -typ und die Kraftaufwendung für die Atemtätigkeit sollte man beurteilen, indem man den Patienten aus kurzer Entfernung beobachtet, um eine Erregung zu vermeiden, die schon allein als solche diese Parameter verändern könnte. Die Atemfrequenz ist im Anschluß an die Geburt oft recht hoch (60 bis 80 Atemzüge/Min.), beträgt aber 1 Stunde post partum in Ruhe durchschnittlich 30 bis 40 Atemzüge/Min. (Rossdale, 1969). Bei hoher Umgebungstemperatur haben Fohlen unter Umständen auch eine erhöhte Atemfrequenz. Diese ist jedoch nicht der einzige Bestimmungsfaktor einer wirkungsvollen Ventilation. Daher sollten die einzelnen Atemzüge hinsichtlich ihrer Tiefe und ihres Charakters eingeschätzt werden, um die Ursache insuffizienter respiratorischer Zustände herauszufinden.

Eine leichte abdominale Komponente der Atemtätigkeit ist normalerweise immer am Ende der Exspirationsphase sowohl in Seitenlage als auch im Stehen zu beobachten, jede merkliche Anstrengung ist aber ein unphysiologischer Befund. Bei einigen Fohlen mit betont abdominalem Kraftaufwand hört man gelegentlich ein Stöhen während der erschwerten und verlängerten Exspiration. Spezielle Erkrankungen wie eine Verlegung der oberen Luftwege oder eine raumbeengende Lungenveränderung verursachen im typischen Fall eine verlängerte tiefe und erschwerte Inspirationsphase bei geblähten Nüstern und übermäßig starker Auswärtsbewegung der Rippen. Ein generalisierter Typ vermehrt angestrengter Atmung kann die Folge eines in erhöhtem Grad hypoxischen Stoffwechsels, einer metabolischen Azidämie und einer Reaktion auf einen unnormal großen mechanischen Widerstand bei der Atmung sein.

Die große Nachgiebigkeit des immaturen Brustkorbs spielt wahrscheinlich eine wichtige Rolle bei der Beeinflussung der Gasaustauscheffizienz der für die Atmung aufgebotenen Kraft. Die respiratorische Insuffizienz bei schwachen frühgeborenen Fohlen geht oft mit Lungenatelektasen in der weichen Lunge einher.

Die liegende Position auf der äußerst wenig Widerstand bietenden Brustwand und die uneffektive Kontrollwirkung von Reflexen auf die Atemtätigkeit üben hierbei den Einfluß aus, mit dessen Hilfe das endexspiratorische Lungenvolumen aufrechterhalten und bei entgegengesetzt wirkenden Kräften gesichert wird. Schwerkranke Fohlen, insbesondere frühgeborene, weisen häufig einen anormalen paradoxen Atemtyp auf, bestehend in Einwärtsbewegung des
Brustkrobs während einer irregulären Inspiration und Dehnung des Abdomens. Diese Art von Verformung der Brustwand macht das Atmen vermehrt kräftezehrend und verschlechtert den Gasaustausch. Mit der Aufrichtung aus der Seiten- in die Brustlage war bei frühgeborenen Fohlen oft ein wesentlicher Rückgang der erforderlichen Anstrengung und Verschwinden der paradoxen Bewegungen festzustellen. Diese Beobachtung war sowohl vom diagnostischen als auch vom therapeutischen Standpunkt aus nützlich.

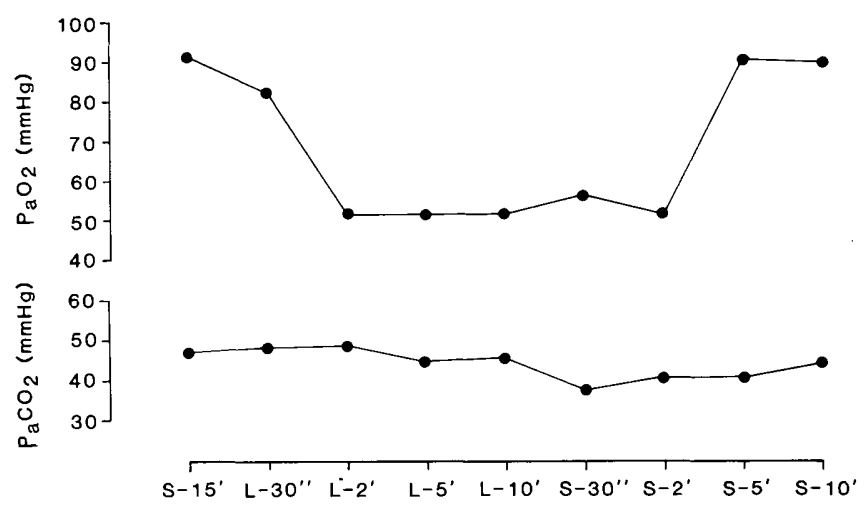

Abb. 1: Einfluß der Körperhaltung auf die arteriellen Blutgaswerte bei einem frühgeborenen Fohlen mit respiratorischer Insuffizienz, das mittels nasaler Insufflation 7 Liter/Min. 100prozentigen Sauerstoff erhielt. Aus einem Arterienverweilkatheter wurden serienmäßig Blutproben entnommen, und zwar nach $15 \mathrm{Min}$. (15'), als das Fohlen ruhig in Brustlage $(S)$ atmete, dann nach Wechsel in Seitenlage ( $L$ ) und darauf nach Wiederaufrichten in Brustlage. Die Zeitdauer jeder Stellung ist entlang der Abszisse aufgetragen. Beachte sowohl den deutlichen Veränderungsgrad als auch den raschen Zeitverlauf bei der ab- bzw. zunehmenden Sauerstoffspannung zwischen den beiden Körperhaltungen.

Zusammen mit der veränderten Körperhaltung, der Brustlage anstelle der Seitenlage, zeigte sich auch ein signifikanter Anstieg des arteriellen Sauerstoffpartialdrucks (Abb.1). Bei dieser Darstellung wurden die Blutproben 15 Minuten nach Legen des Katheters entnommen, dem Zeitpunkt, an dem das Fohlen in Brustlage ruhig atmete. Dann legte man das Fohlen auf die Seite und entnahm Proben nach 30 Sekunden, 2 Minuten, 5 Minuten und 10 Minuten. Nach denselben Zeitintervallen im Anschluß an das Wiederaufrichten in Brustlage wurden erneut Blutproben entnommen. Beim Neugeborenen sind wegen der geringen Körpergröße physiologische Lungengeräusche viel leichter zu hören als beim erwachsenen Tier. Eventuell klingen sie ungewöhnlich laut und scharf, besonders bei erhöhter Atemfrequenz. Die Auskultation des Lungenfeldes ist in vielen Fällen zwar zur Erkennung von Anomalien bei der Atmung erforderlich, die Befunde sind nach der Erfahrung des Autors aber nicht immer positiv korreliert mit der Schwere der Erkrankung. Fohlen, die auskultatorisch wenig oder keine pathologischen Geräusche aufwiesen, litten manchmal unter schweren Lungenerkrankungen, besonders solchen mit Beteiligung des Lungeninterstitiums und Atelektasen, die dann bei der Röntgenuntersuchung oder Sektion zutage traten. Schon nach kurzer Zeit waren bei Seitenlage deutliche Unterschiede zwischen den oben liegenden und den 
unten liegenden Lungenteilen auskultatorisch wahrnehmbar. Dies betraf besonders die Atemgeräusche bei frühgeborenen Fohlen. Wahrscheinlich ist dies eine sekundäre Erscheinung der vermehrten Anfälligkeit der unreifen Lunge dafür, zu kollabieren und atelektatisch zu werden.

Einer eingehenden Untersuchung sollte man bei atmungsinsuffizienten Fohlen das Herz-Kreislauf-System unterziehen. Die physiologische Pulsfrequenz in der Ruhe beträgt zwischen 80 und 120 Pulswellen/Min. Ein holosystolisches Nebengeräusch I. bis III./IV. Grades, am lautesten hörbar an der linken Herzbasis, betrachtet man beim Fohlen im Alter von weniger als 7 Tagen allgemein als normal, und seine Intensität nimmt allmählich ab. Man hält es für ein äußeres Anzeichen des noch vorhandenen Ductus arteriosus (Amoroso, Dawes und Mott, 1958; Rossdale, 1967; Scott, Kneller und Witherspoon. 1975). Erbringt die Auskultation der rechten Seite ein Geräusch und die beider Seiten ein sehr deutliches Geräusch mit oder ohne palpierbares Schwirren und liegt keine schwere Anämie dabei vor, dann läßt dies eine Herzerkrankung vermuten. Weitere diagnostische Maßnahmen wie Echokardiographie, Röntgen oder Kardioangiographie sind indiziert, um einen Herzfehler als das der respiratorischen Insuffizienz zugrundeliegende pathologische Geschehen auszuschließen.

$\mathrm{Ob}$ der Kreislauf funktionsfähig ist, kann mit Hilfe der Pulsqualität und der Kapillarfüllungszeit beurteilt werden. Die Schleimhäute sollten feucht und rosa sein, und die Kapillarfüllungszeit sollte etwa 1 Sekunde betragen. Sind die Schleimhäute nicht zyanotisch, so ist dies nicht notwendigerweise ein Beweis für die ausreichende Sauerstoffversorgung der Gewebe. An der Klinik des Teaching Hospital hatten mehrere atmungsinsuffiziente Fohlen trotz eines $\mathrm{PaO}_{2}$ von etwa $40 \mathrm{mmHg}$ noch eine physiologische Schleimhautfarbe. An den Schleimhäuten sichtbare Petechien sind im allgemeinen Symptome einer disseminierten intravasalen Blutgerinnung, einer Septikämie und einer Asphyxie. Eine leichte Gelbfärbung ist ein häufig erhobener, aber unspezifischer Befund bei vielen kranken Fohlen; schwerer Ikterus weist auf Leberversagen oder neonatale Hämolyse hin. Im letzteren Fall kann eine schwere Anämie auch zur erhöhten Atemfrequenz beitragen. Schwache, unreife Fohlen sollten hinsichtlich der Erkrankungen des Atmungsapparates als Risikopatienten betrachtet werden. Diese Fohlen sollen hinsichtlich Kräftigkeit, Geisteszustand und Entwicklungsgrad beurteilt werden. Weiche biegsame Ohren, ein seidiges kurzes Haarkleid, Beugesehnenschwäche und niedriges Geburtsgewicht deuten auf eine Frühgeburt oder Geburtsunreife hin. Die Haut, der mediale Augenwinkel und die Innenseite der Nüstern sollten auf eine eventuell sichtbare Verfärbung durch Mekonium untersucht werden, was die Diagnose Mekoniumaspiration stützen würde.

Die meisten Störungen des Atmungsapparates bei Neugeborenen führen $\mathrm{zu}$ ähnlichen klinischen Erscheinungen, und die klinische Untersuchung leistet daher wertvolle Dienste bei der Erkennung der Erkrankung und ihrer Schwere, gewöhnlich nicht aber beim Auffinden der Ursache. Manchmal gibt die den Geburtszeitraum betreffende Anamnese einen Hinweis auf die Ätiologie, aber zur Dia- gnose und Behandlung benötigt man in vielen Fällen die zusätzlichen Ergebnisse der Labor- und Röntgenuntersuchung.

\section{Klinische Pathologie}

Rote und weiße Blutzellen sollten regelmäßig gezählt und ein weißes Blutbild ausdifferenziert werden. Man sollte möglichst früh Infektionserreger zu identifizieren versuchen. Fohlen mit Septikämie zeigen meist eine Neutropenie, die bei Tieren, welche die akute Phase der Erkrankung überstehen, oft in eine Neutrophilie umschlägt. Bei 90 Prozent der Fohlen mit Septikämie ergab die Zählung der neutrophilen Leukozyten auf dem Teststreifen Werte über $0,2 \cdot 10^{\circ} /$ Liter und toxische Veränderungen an den neutrophilen Zellen (toxisch bedingte Granulation, Doehle-Körper) (Koterba et al., 1984). Diese Befunde werden jedoch auch häufig bei Fohlen erhoben, bei denen die angelegten Blutkulturen ein negatives Ergebnis liefern, die aber irgendeine Streßsituation, insbesondere Asphyxie, durchgemacht haben. Ein erhöhter Fibrinogengehalt (über 4,0g/Liter) am ersten Tag nach der Geburt legt den dringenden Verdacht nahe, daß sich das Fohlen in utero eine Infektion zugezogen hat. Der Zinksulfat-Trübungstest, der einen Wert unter 8,0 g/Liter liefert, kennzeichnet Fohlen mit Hypogammaglobulinämie (Koterba et al., 1984). Blutkulturen sollte man von jedem Fohlen mit respiratorischer Insuffizienz anlegen, da sie als Beweis einer Infektion Grundlage zielgerichteter Therapie sind.

Im Fall einer Infektion zeigt Blut, das nach chirurgischem Eingehen durch die Haut aus der Jugular- oder Medianvene entnommen und mit den Nährböden für anaerobe und aerobe Kulturen beschickt wurde, innerhalb von 24 Stunden ein sichtbares Wachstum. Die mikroskopische Untersuchung eines gefärbten Blutausstrichs leistet unter Umständen wertvolle Dienste, da sie, früh genug eingeleitet, den Nachweis für eine Bakteriämie liefert. Es ist auch möglich, eine Trachealspülung durchzuführen, um Material für eine Kultur und zur zytologischen Untersuchung zu gewinnen. Die erforderlichen Fixierungsmaßnahmen sollten aber nicht mit zuviel Streß für das Tier verbunden sein.

\section{Spezielle Diagnosemethoden}

\section{Röntgen des Thorax}

Die Röntgenuntersuchung des Thorax ist oft zur Bestätigung einer Erkrankung des Atmungsapparats erforderlich. Zudem sagt sie aus, wieviel Lungengewebe krankhaft verändert ist. An der Universitätsklinik bestand bei mehreren Fohlen nicht einmal der Verdacht einer Beteiligung der Lunge am pathologischen Geschehen, bis dann routinemäßig Röntgenaufnahmen vom Thorax gemacht und Veränderungen entdeckt wurden.

Der Erfolg der Röntgenuntersuchung hängt davon ab, daß man unphysiologische Verschattungsmuster erkennt und Listen von differentialdiagnostischen Möglichkeiten erstellt, mit Hilfe deren man die sich auf dem Röntgenbild zeigenden Anomalien interpretieren kann. Thoraxaufnahmen sind in der Humanmedizin ein besonders nützlicher Untersuchungsbestandteil, und verschiedene spezielle $\mathrm{Mu}$ - 


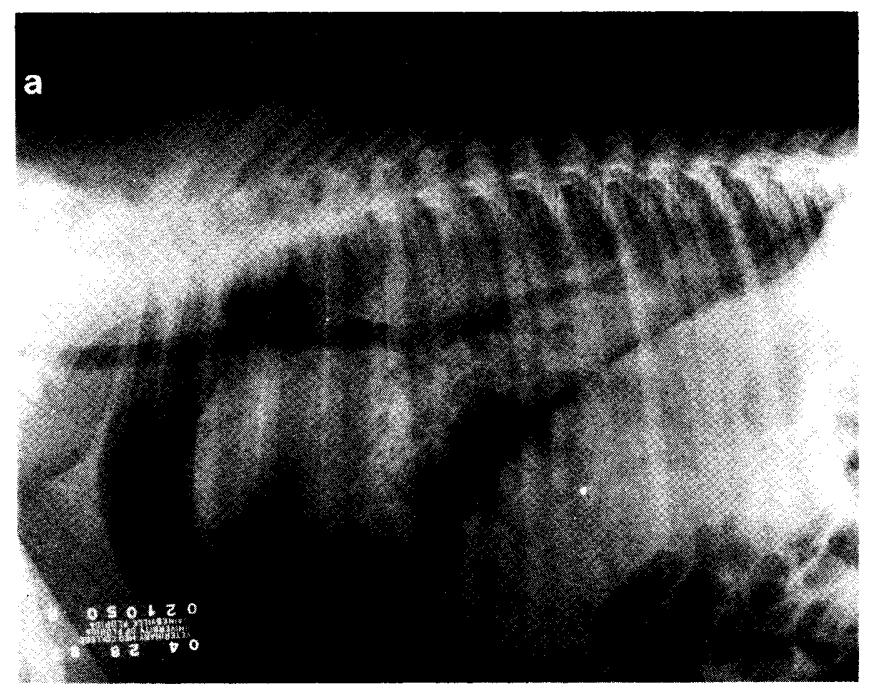

Abb. 2: (a) Röntgenaufnahme des Thorax eines sechs Tage alten Vollblutfohlens im Zustand respiratorischer Insuffizienz mit schwerer Pneumonie, die sekundär zu einer Staphylococcus-aureus-Septikämie aufgetreten war. (b) Dasselbe Fohlen fünf Tage später, als sich das klinische Erscheinungsbild wesentlich gebessert hatte. Bemerkenswert ist die nahezu vollständige Auflösung des zuvor ausgedehnten Lungeninfiltrats.

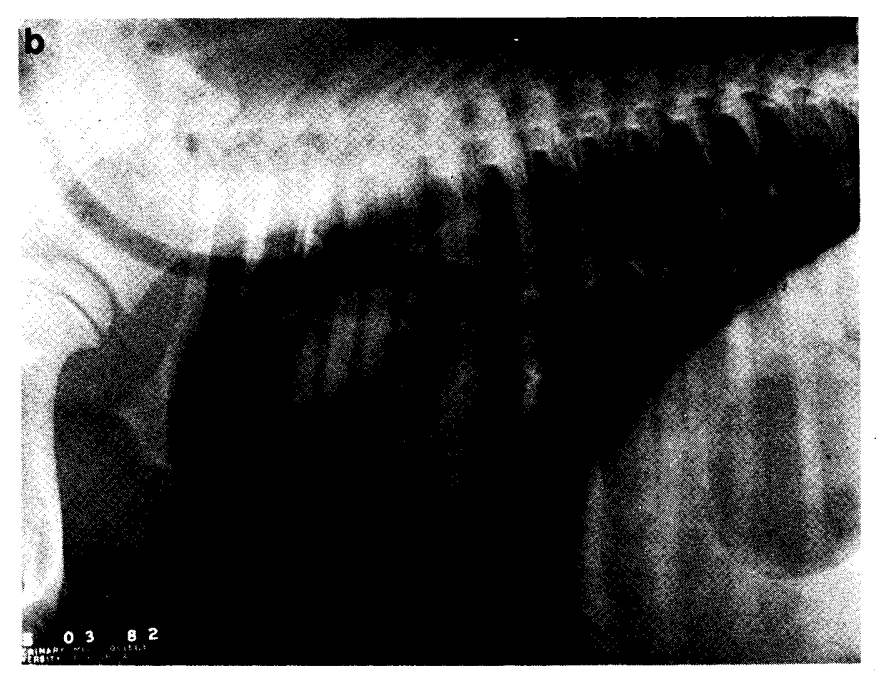

ster haben sich als charakteristisch für bestimmte Krankheitsprozesse erwiesen. Die Membranerkrankung der Früh- und Neugeborenen stellt sich zum Beispiel in ihrer typischen Form als milchglasartiges oder granuliertes Lungenfeld mit darin hervortretendem luftgefülltem Bronchogramm dar. Weil die Röntgenuntersuchung des Thorax beim neugeborenen Pferd bisher nicht routinemäßig durchgeführt wurde, ist auch der Bereich physiologischer Befunde noch nicht ausreichend abgegrenzt, und die Beurteilung bewegt sich noch im Entwicklungsstadium. Der Zustand des Atmungsapparats kann sich beim Neugeborenen, verglichen mit dem erwachsenen Tier, rasch ändern. Die Röntgenuntersuchung bietet somit eine gute Möglichkeit, den Stand der Vorgänge zu überwachen. Der Fall in Abb. 2 demonstriert dies. Bei einem Stutfohlen kam es nach 320 Tagen Trächtigkeitsdauer zur Staphylococcusaureus-Septikämie, zur schweren Pneumonie und zur At- mungsinsuffizienz. Diese Erscheinungen sprachen rasch auf die antibiotische Therapie an, und nach 10tägiger Sauerstoffapplikation und Überwachung durch einen Pfleger trat vollständige Genesung ein.

\section{Arterielle Blutgasspannungen}

Wenn die unterstützende Beatmung wirkungsvoll und mit der gebotenen Sicherheit über längere Zeit durchgeführt werden soll, müssen die Blutgase überwacht werden. Zusammen mit den klinischen Untersuchungsbefunden und den übrigen Laborwerten kann man mit Hilfe der Blutgasdaten die Schwere der Atemwegserkrankung und die Art der erforderlichen Behandlung bestimmen, ebenso die Reaktion des einzelnen Patienten auf diese Behandlung. Leider haben Tierärzte in der Vergangenheit die arterielle Blutgasanalyse nach Möglichkeit umgangen, weil die benötigte Ausrüstung teuer ist, Labors mit der erforderlichen Ausstattung nicht günstig zur Klinik gelegen sind und irrtümlicherweise angenommen wird, die Probenentnahme sei zu schwierig und die Ergebnisse seien nicht leicht $\mathrm{zu}$ interpretieren. Wenn aber der Sauerstoffpartialdruck in den Alveolen über einen ausreichend langen Zeitraum hinweg auf einem hohen Niveau gehalten wird, können bei Mensch und Tier Resorptionsatelektase und Sauerstoffvergiftung die Folge sein (West, 1982).

Eine aussagekräftige Interpretation der arteriellen Blutgaswerte hängt nicht nur von der korrekten Probenentnahmetechnik, sondern auch von der Körperhaltung des Fohlens, wie stark es strampelt und von der Sauerstoffkonzentration der eingeatmeten Luft zum Zeitpunkt der Probenentnahme ab. Strampeln während der Probenentnahme führt gewöhnlich zu einem vorübergehenden, aber deutlichen Abfall der arteriellen Kohlendioxidspannung $\left(\mathrm{PaCO}_{2}\right)$. Bei dem in Abb. 1 untersuchten Fohlen hatte das Wiederaufrichten von Seiten- in Brustlage zur Folge, daß sich das Fohlen erregte und der $\mathrm{PaCO}_{2}$ von $44,9 \mathrm{mmHg}$ auf $37,2 \mathrm{mmHg}$ abfiel. Der $\mathrm{PaO}_{2}$ wird ebenfalls von außen durch die anteilsmäßige Konzentration des eingeatmeten Sauerstoffes $\left(\mathrm{FI}_{2} \mathrm{O}_{2}\right)$ beeinflußt, und er steigt in unterschiedlichem Maße je nach Vorhandensein und Größe von Rechts-links-Shunts.

Trächtigkeitszeitpunkt und Alter des Fohlens, von der Geburt an gerechnet, üben Einfluß auf die physiologischen Werte aus. Nach der Geburt besteht zu einem gewissen Grad eine Hypoxämie und eine respiratorische Azidose (ausgehend von den Normalwerten beim erwachsenen Tier), aber beide dauern normalerweise nicht länger an als bis zum Alter von 12 bis 24 Stunden bei ausgetragenen und etwas länger bei frühgeborenen Fohlen (Rose, Rossdale und Leadon, 1982). Die Blutgaswerte der Tabelle 2 stammen von Rossdale und Mitarbeitern, gemessen bei nach normaler Trächtigkeitsdauer zur Welt gekommenen und frühgeborenen Fohlen, und zwar die Daten für $\mathrm{PaO}_{2}, \mathrm{PaCO}_{2}$ und $\mathrm{pH}$; berechnet wurden die 95-\%-Konfidenzgrenzen, wobei man von einer Normalverteilung der Meßwerte ausging. Ihrer Erfahrung gemäß würden die genannten Autoren eine Behandlung bei Fohlen, besonders wenn sie älter als 48 Stunden sind, mit einem $\mathrm{PaO}_{2}$ unter $60 \mathrm{~mm} \mathrm{Hg}$ oder einem $\mathrm{PaCO}_{2}$ über $50 \mathrm{~mm} \mathrm{Hg}$ in Betracht ziehen. 


\begin{tabular}{|c|c|c|c|c|c|c|c|c|c|c|}
\hline \multirow[b]{2}{*}{$\begin{array}{l}\text { Geburts- } \\
\text { termin }\end{array}$} & \multicolumn{3}{|c|}{ Fohlen } & \multicolumn{2}{|c|}{$\mathrm{PaO}_{2}$} & \multicolumn{2}{|c|}{$\mathrm{PaCO}_{2}$} & \multicolumn{2}{|c|}{$\mathrm{pH}$} & \multirow[t]{2}{*}{ Literatur } \\
\hline & & $\begin{array}{l}\text { Alter } \\
\text { (h) }\end{array}$ & Anzahl & $\begin{array}{l}\text { Mittelwert } \\
( \pm \text { Stan- } \\
\text { dardab- } \\
\text { weichung) }\end{array}$ & $\begin{array}{l}\text { 95-\%-Kon- } \\
\text { fidenz- } \\
\text { grenzen }\end{array}$ & $\begin{array}{l}\text { Mittelwert } \\
( \pm \text { Stan- } \\
\text { dardab- } \\
\text { weichung) }\end{array}$ & $\begin{array}{l}\text { 95-\%-Kon- } \\
\text { fidenz- } \\
\text { grenzen }\end{array}$ & $\begin{array}{l}\text { Mittelwert } \\
\text { ( } \pm \text { Stan- } \\
\text { dardab- } \\
\text { weichung) }\end{array}$ & $\begin{array}{l}\text { 95-\%-Kon- } \\
\text { fidenz- } \\
\text { grenzen }\end{array}$ & \\
\hline normal & 3 & -168 & 10 & $80,1 \pm 3,8$ & $56-104$ & $47,5 \pm 2,6$ & $31-62$ & $7,354 \pm 0,011$ & $7,28-7,42$ & $\begin{array}{l}\text { Rossdale } \\
(1970)\end{array}$ \\
\hline $\begin{array}{l}\text { normal } \\
\text { normal } \\
\text { normal } \\
\text { normal } \\
\text { Frühgeburt }\end{array}$ & $\begin{array}{c}1 \\
12 \\
48 \\
4 \\
0,5\end{array}$ & $\begin{array}{r}-12 \\
-48 \\
-168 \\
-11 \\
5-11\end{array}$ & $\begin{array}{l}6 \\
6 \\
5 \\
5 \\
7\end{array}$ & $\begin{array}{l}77,4 \pm 3,1 \\
83,2 \pm 3,1 \\
88,2 \pm 5,9 \\
83,8 \pm 6,3 \\
53,7 \pm 1,5\end{array}$ & $\begin{array}{l}60-92 \\
68-98 \\
61-114 \\
55-111 \\
45-62\end{array}$ & $\begin{array}{l}42,2 \pm 1,8 \\
44,5 \pm 1,2 \\
42,4 \pm 1,0 \\
39,5 \pm 1,8 \\
55,3 \pm 3,6\end{array}$ & $\begin{array}{l}34-50 \\
38-50 \\
37-47 \\
31-47 \\
36-74\end{array}$ & $\begin{array}{l}7,378 \pm 0,015 \\
7,374 \pm 0,004 \\
7,384 \pm 0,014 \\
7,367 \pm 0,010 \\
7,208 \pm 0,048\end{array}$ & $\left.\begin{array}{l}7,34-7,41 \\
7,35-7,39 \\
7,32-7,45\end{array}\right\}$ & $\begin{array}{l}\text { Rose et al. } \\
(1982) \\
\text { Rose et al. } \\
(1983)\end{array}$ \\
\hline
\end{tabular}

Tab. 2: Blutgaswerte bei ausgetragenen und frühgeborenen Fohlen

\section{Hypoxämie}

Die Ursachen eines unnormal niedrigen $\mathrm{PaO}_{2}$ oder einer Hypoxämie (unter $60 \mathrm{~mm} \mathrm{Hg}$ ) sind nach der Häufigkeit ihres Vorkommens ansteigend geordnet: (1) niedrigerer Sauerstoffgehalt der Inspirationsluft, (2) beeinträchtigte Diffusion, (3) unzureichende Ventilation der Alveolen (Hypoventilation), (4) Mißverhältnis zwischen Ventilation und Perfusion und/oder (5) Gefäßshunts von der rechten zur linken Kreislaufhälfte.

Die ersten beiden oben angeführten Gründe kommen als primäre Verursacher einer Hypoxie kaum vor. Erscheinung (3) führt nicht nur zur Hypoxämie, sondern auch zur Hyperkapnie $\left(\mathrm{PaCO}_{2}\right.$ über $\left.50 \mathrm{~mm} \mathrm{Hg}\right)$. Die Hypoventilation ist die Folge eines Verstopfung oder Einschnürung bewirkenden Prozesses, bei dem das Fohlen den erhöhten Widerstand bei der Atmung nicht kompensieren kann, oder einer neurologischen Funktionsstörung, z. B. einer Schädigung des Zentralnervensystems. Die Beimengung von venösem Blut als Resultat eines Mißverhältnisses oder Shunts führt im typischen Fall zu einem sehr niedrigen $\mathrm{PaO}_{2}$. Gewöhnlich geht dies mit einer deutlichen Zunahme der Atemfrequenz und der für die Atmung aufgewendeten Kraftanstrengung einher, da die peripheren Chemorezeptoren stimuliert werden. In vielen Fällen sprechen Fohlen mit einem Mißverhältnis zwischen Ventilation und Perfusion gut auf die Sauerstofftherapie an, andere jedoch trotz sehr hoher Sauerstoffkonzentration der Inspirationsluft nicht. Die ausbleibende Reaktion rührt von vorhandenen großen Shunts her, die durch ausgedehnte Lungenatelektasen, Gewebskonsolidierung oder einen persistierenden fötalen Kreislauf verursacht sind. Man hat Vergleiche hinsichtlich der günstigen diagnostischen und prognostischen Auswirkungen zwischen der Reaktion ausgetragener, frühgeborener und anormaler Fohlen auf nasale Sauerstoffinsufflation von 10 Litern/Min. (Rose, Hodgson, Leadon und Rossdale, 1983) und derjenigen auf 100prozentigen über eine Gesichtsmaske verabreichten Sauerstoff (Rossdale, 1970) angestellt. Dabei zeigte sich, daß beide Verfahren zur Sauerstoffapplikation den $\mathrm{PaO}_{2}$ etwa gleich stark in Bereiche von etwa $200 \mathrm{~mm} \mathrm{Hg}$ bei ausgetragenen Fohlen, verglichen mit $160 \mathrm{~mm} \mathrm{Hg}$ oder darunter bei frühgeborenen und kranken Fohlen, anheben.

\section{Hyperkapnie}

In vielen Fällen ist der $\mathrm{PaCO}_{2}$ bei hypoxämischen Fohlen normal oder niedrig, weil das Fohlen sogar schwer erkrankte Lungen beim Bestreben, die Hypoxämie zu kompensieren, zu ventilieren vermag. So kann sich bei Fohlen

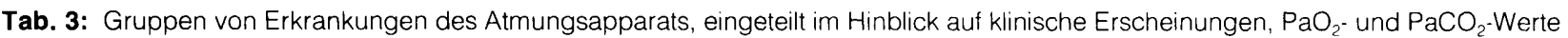

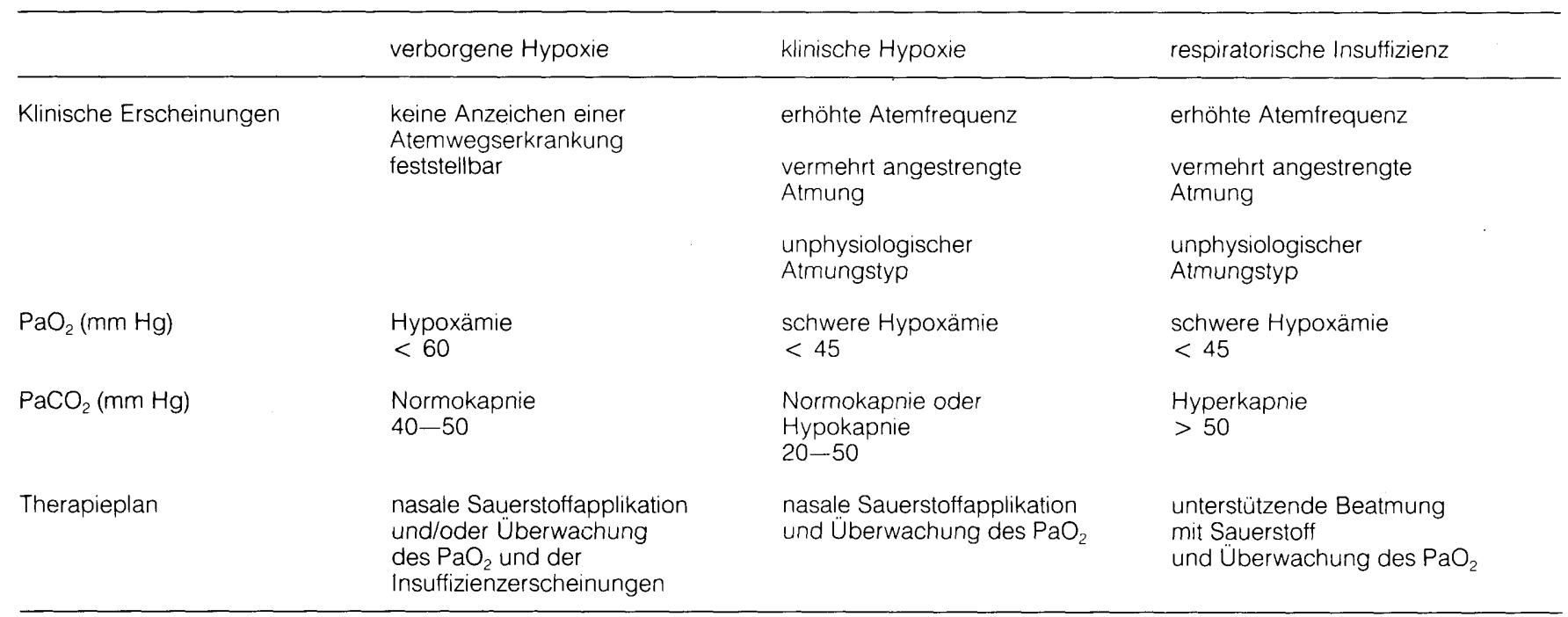


als Reaktion auf eine schwere Hypoxämie Hopykapnie einstellen. Die Hypokapnie bei hypoxämischen Fohlen weist auf eine primäre Depression der zentralen Atemregulationsmechanismen hin (Schädigung des Zentralnervensystems, Arzneimittel, Vergiftung) oder auf eine respiratorische Dekompensation in späteren Stadien einer schweren, eine Verlegung oder Einengung der Atemwege verursachenden Störung hin. Wenn der mechanische Widerstand bei der Atmung die metabolischen Reservekapazitäten eines kranken Fohlens übersteigt, kommt es direkt zur Atmungsinsuffizienz, der ausschließlich mit Hilfe einer unterstützenden Beatmung entgegengewirkt werden kann.

\section{Klinische Beobachtungen \\ im Zusammenhang mit Blutgasanomalien}

Die Autoren haben auf der Grundlage einer gemeinsamen Betrachtung der Blutgaswerte eines Individuums und der klinischen Stadien, welche Fohlen mit einer Erkrankung des Atmungsapparats durchlaufen, drei Kategorien beobachtet (Tabelle3).

Bei Fohlen, die sich in den ersten Stunden oder Tagen nach der Geburt nur schwer adaptierten, diagnostizierte man eine „verborgene Hypoxie“. Die Tiere zeigten abnormes Verhalten, aber keine Anzeichen einer Erkrankung des Atmungsapparats. Die arterielle Blutgasanalyse ergab eine leichte bis mäßige Hypoxämie $\left(\mathrm{PaO}_{2}\right.$ unter $\left.60 \mathrm{~mm} \mathrm{Hg}\right)$ bei Normokapnie. Die Befunde der klinischen Untersuchung waren oft unauffällig, die Röntgenuntersuchung des Thorax zeigte aber das Bild einer in gewissem Grade ablaufenden Lungenerkrankung. Bei dieser Gruppe wurde nasale Sauerstoffinsufflation angewandt, ausreichend, um den arteriellen Sauerstoffpartialdruck auf 80 bis $100 \mathrm{~mm} \mathrm{Hg}$ anzuheben und bis das Fohlen so lebhaft war, daß keine weitere Sauerstofftherapie mehr möglich war.

„Klinische Hypoxie“ und respiratorische Insuffizienz stellte man bei Fohlen fest, die klare Anzeichen der Atmungsinsuffizienz aufwiesen. Diese Fohlen hatten meist eine deutlich erhöhte Atemfrequenz mit vermehrt angestrengter Atemtätigkeit. Zu den ungewöhnlichen Bewegungen bei der Atmung gehörten Einwärtsbewegung der Rippen, deutliche abdominale Kraftanstrengung mit Keuchen bei der Exspiration und paradoxe Verformung der Brustwand. Trotz ähnlichem klinischem Erscheinungsbild ergab die Blutgasanalyse zwei verschiedene Bilder aus den jeweiligen Werten eines Individuums. Bei „klinischer Hypoxie“ wies die Blutgasanalyse auf eine schwere Hypoxämie $\left(\mathrm{PaO}_{2}\right.$ unter $45 \mathrm{~mm} \mathrm{Hg}$ ) bei unterschiedlichen $\mathrm{PaCO}_{2}$-Werten $(20$ bis $50 \mathrm{~mm} \mathrm{Hg}$ ) hin. Der Ernst des pathologischen Geschehens war aus der Auskultation und der Röntgenuntersuchung des Thorax klar ersichtlich; dennoch waren diese Fohlen noch kräftig genug, um die Lunge zumindest in den Anfangsstadien der Erkrankung angemessen zu ventilieren, manchmal sogar als Reaktion auf die schwere Hypoxämie zu hyperventilieren. Die Herabsetzung der Atemfrequenz und des Kraftaufwandes für die Atmung direkt nach Beginn der Sauerstofftherapie stand in Übereinstimmung mit der Vermutung, daß zumindest ein Teil der Insuffizienz durch die hypoxische Stoffwechsellage verursacht war. Die Reaktion auf die Sauerstoffanreicherung variierte in Ab- hängigkeit von der Größe der vorhandenen Shunts. Bei großen Shuntverbindungen sind allgemein unter Umständen sehr hohe $\mathrm{FI}_{2} \mathrm{O}_{2}$-Niveaus erforderlich, um den $\mathrm{PaO}_{2}$ befriedigend anzuheben.

Fohlen mit respiratorischer Insuffizienz hatten, wie sich zeigte, eine schwere Hypoxämie $\left(\mathrm{PaO}_{2}\right.$ unter $\left.45 \mathrm{~mm} \mathrm{Hg}\right)$, Hyperkapnie $\left(\mathrm{PaCO}_{2}\right.$ über $\left.50 \mathrm{~mm} \mathrm{Hg}\right)$ und Azidämie mit respiratorischer und metabolischer Komponente. Einige dieser Fohlen hatten eine zentralnervöse Schädigung erlitten, eine Vergiftung oder eine periodische Frühgeborenenapnoe durchgemacht. Andere litten an einer niederdrükkenden Pneumonie oder einer anderen fortschreitenden Atemwegserkrankung, die schließlich zu einer Ermüdung der Atemmuskulatur führte und das Fohlen unfähig werden ließ, die Ventilation der Alveolen aufrechtzuerhalten. Die respiratorische Dekompensation setzte bei diesen Fohlen normalerweise rasch ein und zog trotz erhöhter Atemfrequenz und deutlicher Kraftanstrengung beim Atmen Kohlendioxidretention und Azidämie nach sich.

Zum Überleben war unterstützende Beatmung erforderlich.

\section{Sauerstofflu $\beta$}

Die Abschätzung des Sauerstoffflusses bei einem Fohlen mit Erkrankung des Atmungsapparats ist sehr wichtig. Unzureichender Gasaustausch in der Lunge und daraus resultierende arterielle Hypoxämie ist die häufigste, aber nicht die einzige Ursache der Hypoxie beim Fohlen. Die Sauerstoffversorgung der Gewebe hängt letzten Endes von der Sauerstoffmenge ab, die mit dem Blut ins Gewebe gelangt. Die Sauerstoffbelieferung ist das Produkt der arteriellen Sauerstoffkonzentration und des Herzminutenvolumens. Daher können schwere Anämie, großer Blutverlust oder Herzversagen durch unzureichende Sauerstoffzulieferung an die Körpergewebe eine Hypoxie hervorrufen. Ein weiterer möglicher Grund für die Hypoxie ist eine zytotoxische Schädigung der Mechanismen der Zellatmung, der Grundlage der normalen Verstoffwechselung von Sauerstoff im Gewebe. Man muß sich also im klaren darüber sein, daß Fohlen, deren Hypoxie durch ungenügenden Sauerstofftransport oder ungenügende Verwertung verursacht wird, nicht auf eine Sauerstofftherapie ansprechen, da mit einer Anhebung des $\mathrm{PaO}_{2}$ auf über $80 \mathrm{~mm} \mathrm{Hg}$ die arterielle Sauerstoffkonzentration kaum beeinflußt wird.

\section{Verfabren zur Probenentnabme von Arterienblut und zur Überwachung der Blutgasspannungen}

Arterienblutproben sind direkt mittels Punktion aus der Carotis, der Brachial-, der großen Metatarsalarterie oder der Arteria facialis zu entnehmen (Rose und Rossdale, 1981). Bei widerspenstigen Patienten kann man sich die kunstgerechte Probenentnahme wesentlich erleichtern, indem man ein kleines Depot eines Lokalanästhetikums in der Haut über der Arterie setzt. Auf diese Weise sind unerwünschte Abwehrbewegungen, die zur Hyperventilation führen, weitgehend zu verhindern. Kann eine Probe nicht sofort untersucht werden, so verschließt man die Spritze mit dem Probenblut luftdicht und bewahrt sie in Eis auf; der $\mathrm{pH}$ 
verändert sich 3,5 Stunden lang und $\mathrm{PaO}_{2}$ und $\mathrm{PaCO}_{2}$ verändern sich 6 Stunden lang nicht wesentlich (Haskins, 1977).

Bei einem schwer erkrankten Fohlen mit schlechter Durchblutung ist der Arterienpuls unter Umständen so schwach, daß die Arterienpunktion schwierig ist. Dann kann man mit Hilfe der Gasanalyse von Venenblut in Verbindung mit dem klinischen Erscheinungsbild die Schwere der Erkrankung abschätzen. Venenblutproben sollten aus einer großen Vene mit freiem Blutfluß (z. B. der Jugularvene) entnommen werden, um Verfälschungen, die durch Probenentnahme in einem bestimmten Körperbereich entstehen, möglichst gering zu halten. Die Ergebnisse kann man zur Bewertung der Stoffwechselsituation heranziehen und um eine Orientierung in der Frage zu erhalten, ob versucht werden sollte, mit weitergehenden invasiven Methoden eine Arterienblutprobe zu bekommen. Ist der Jugularvenen- $\mathrm{PaCO}_{2}$ höher als $60 \mathrm{~mm} \mathrm{Hg}$, so besteht wahrscheinlich eine arterielle Hyperkapnie, und ein venöser $\mathrm{PaO}_{2}$ von unter $20 \mathrm{~mm} \mathrm{Hg}$ läßt eine arterielle Hypoxämie oder ein Herzversagen vermuten.

Zur exakten Beurteilung und Überwachung des Gasaustauschs in der Lunge sind serienmäßige arterielle Blutgasanalysen erforderlich. Schwierigkeiten mit der Duldsamkeit des Patienten und Gefäßtraumatisierung nach häufiger Punktierung können der Durchführung einer genauen Überwachung jedoch kaum überwindbare Grenzen setzen. Das Schieben eines Arterien-Verweilkatheters unter Lokalanästhesie durch die Haut hindurch ist nicht schwierig, vorausgesetzt die Pulsqualität ist ausreichend. Probleme gibt es dagegen beim Offenhalten und Halten des Katheters in seiner Lage bei allen Patienten außer denen in äußerst schlechter Verfassung. Ein invasives Verfahren ist, unter kurzer Inhalationsanästhesie über der großen Metatarsalarterie chirurgisch einzugehen und einen humanmedizinischen Säuglings-Umbilikalkatheter zu setzen. Solche Katheter bleiben aber nur für eine begrenzte Zeit (12 bis 48 Stunden) durchgängig, wenn sie zwischendurch mit heparinisierter Kochsalzlösung gespült werden. Man muß ununterbrochen langsam Kochsalzlösung infundieren, da Arterienblutproben in den meisten Fällen über einen längeren Zeitraum hinweg benötigt werden.

In letzter Zeit sind nichtinvasive Meßverfahren der Gewebssauerstoffversorgung erprobt worden. Transkutane Sauerstoffüberwachungsgeräte sind bei menschlichen Säuglingen weitverbreitet in Anwendung. War die periphere Durchblutung ausreichend, hat sich eine ausgezeichnete

\section{Literatur}

Avery, M. E., Fletcher, B. D., und Williams, R. G. (1981): The Lung and Its Disorders in the Newborn Infant, 4 th edn. W. B. Saunders, Philadelphia.

Amoroso, E. C., Dawes, G. S., und Mott, J. C. (1958): Patency of the ductus arteriosus in the newborn calf and foal. Br. Heart J. 20, 92-96.

Crouch, G. M., und Morgan, S. J. (1983): Bilateral choanal atresia in a foal. Comp. Cont. Educ. 5, 206-211.

Dubielzig, R. R. (1977): Pulmonary lesions of neonatal foals. J. Equine Med. Surg. 1, 419-425.

Gregory, G. (1972): Respiratory care of newborn infants. Pediatr. Clin. Am. 19, 311.
Übereinstimmung mit arteriellen Blutgaswerten gefunden. In einer Gruppe fünf Tage alter Fohlen wurden transkutane Überwachungsgeräte zweier Hersteller benützt, aber keines der Geräte lieferte, wie sich herausstellte, längere Zeit brauchbare Daten (Warren, Webb, Kosch und Coons, 1984). Ein Apparat, der eine polarographische Miniaturelektrode besitzt, die unter dem Augenlid des Fohlens an die Konjunktiva angesetzt wird, und an dem ununterbrochen die Sauerstoffspannung abgelesen werden kann, wurde einer Bewertung unterzogen, und erste Ergebnisse waren vielversprechend (Webb, Danile, Miller und Kosch, 1984b). Ein großer Vorzug der Anwendung direkter Verfahren ist, daß man die Gewebssauerstoffversorgung fortlaufend überprüfen und so Veränderungen des Sauerstoffgehalts schon im Anfangsstadium erkennen kann.

\section{Schlußfolgerungen}

Die Grundüberlegungen, die in dieser Veröffentlichung ausgeführt wurden, gewähren Tierärzten, die mit der Diagnose und Beurteilung von Fohlen mit respiratorischer Insuffizienz befaßt wird, neue Einblicke und neue Orientierungsrichtlinien. Verfahren wie die sorgfältige klinische Überwachung von Beatmungsmaßnahmen, Röntgenuntersuchung des Thorax, Anlegen von Blutkulturen und arterielle oder indirekte Blutgasanalyse erweisen sich eventuell als nützlich. Es sind Methoden, die es dem Kliniker ermöglichen, Art und Schwere einer Erkrankung des Atmungsapparats früher zu erkennen und besser die Bedürfnisse dieser Fohlen hinsichtlich der Therapie abzuschätzen.

Die Autoren danken der Equine Neonatology Study Group der Universität Florida und der Florida Thoroughbred Breeders' Association für ihre finanzielle Unterstützung und allen Studenten, dem Lehrkörper und dem Personal für ihre Bemühungen; sie alle haben in den vergangenen drei Jahren geholfen, die klinische Überwachung von Fohlen, die in das Veterinary Medical Teaching Hospital eingeliefert wurden, zu verbessern. Dieser Artikel wird veröffentlicht als Florida Agricultural Experiment Station Journal,

Ausgaben-Nummer 5090.

\section{P. C. Kosch,}

Departments of Medical Sciences and Physiological Sciences,

College of Veterinary Medicine,

University of Florida,

Gainesville,

Florida 32610, USA

Erschienen in Equine Veterinary Journal (1984) 16 (4) 312-318.

Übersetzt und veröffentlicht mit freundlicher Genehmigung der British Equine Veterinary Association.

Haskins, S. C. (1977): Sampling and storage of blood for $\mathrm{pH}$ and blood gas analysis. J. Am. vet. med. Ass. 170, 429-433.

Klaus, M., Fanaroff, A., und Martin, R. J. (1979): Respiratory problems. In: Care of the High Risk Neonate, 2nd. edn. Eds M. H. Klaus and A. A. Fanaroff. W. B. Saunders, Philadelphia.

Koterba, A. M., Brewer, B. D., und Tarplee, F. A. (1984): Clinical and clinicopathological characteristics or septicaemic neonatal foals: A review of 38 cases. Equine vet. J. 16, 376-382.

Martens, R. J. (1982): Neonatal respiratory distress: A review with emphasis on the horse. Comp. Cont. Educ. 4, 23-33.

Merkt, H. S., und von Lepel, J. D. (1971): Report on the Autumn $1970 \mathrm{ex}-$ aminations of West German Thoroughbreds (bloodstock). Vollblut, Zucht und Rennen 34, 26-30. 
Pattle, R. E., Rossdale, P. D., Schock, C., und Creasey, J. M. (1975): The development of the lung and its surfactant in the foal and in other species. J. Reprod. Fert. Suppl. 23, 651-657.

Platt, H. (1973): Aetiological aspects of perinatal mortality in the Thoroughbred. Equine vet. J. 5, 116-120.

Rose, R. J., und Rossdale, P. D. (1981): Techniques and clinical applications of arterial blood collection in the horse. Equine vet. J. 13, 70-73.

Rose, R. J., Rossdale, P. D., und Leadon, D. P. (1982): Blood gas and acidbase status in spontaneously delivered, term-induced, and induced premature foals. J. Reprod. Fert. Suppl. 32, 521-528.

Rose, R. J., Hodgson, D. R., Leadon, D. P., und Rossdale, P. D. (1983): Effect of intranasal oxygen administration on arterial blood gas and acid base parameters in spontaneously delivered, term-induced, and induced premature foals. Res. vet. Sci. 34, 159-162.

Rossdale, P. D. (1967): Clinical studies on the newborn Thoroughbred foal. II. Heart rate, auscultation and electrocardiogram. Br. vet. J. 123, $521-532$.

Rossdale, P. D. (1969): Measurements of pulmonary ventilation in normal newborn Thoroughbred foals during the first three days of life. Br. vet. J. 125, 157.

Rossdale, P. D. (1970): Some parameters of respiratory function in normal and abnormal foals with special reference to levels of $\mathrm{pO}_{2}$ during air and oxygen inhalation. Res. vet. Sci. 11, 270-276.

Rossdale, P. D. (1979): Neonatal respiratory problems of foals. Vet. Clin. N. Am. Lg. Anim. Pract. 1, 205-217.
Rossdale, P. D. (1980): Neonatal problems in the horse. In: Current Therapy in Theriogenology. Ed. D. A. Morrow, Saunders, Philadelphia. 755-767.

Rossdale, P. D., und Leadon, D. (1975): Equine neonatal disease: A review. J. Reprod. Fert. Suppl. 23, 685-690.

Rossdale, P. D., Pattle, R. E., und Mahaffey, L. W. (1967): Respiratory distress in a newborn roal with failure to form lung lining film. Nature 215, 1498-1499.

Rossdale, P. D., und Ricketts, S. W. (1980): The adaptive period. In: Equine Stud Farm Medicine. 2nd edn. Bailliere Tindall, London.

Scott, E. A., Kneller, S. K., und Witherspoon, D. M. (1975): Closure of ductus arteriosus determined by cardiac catheterization and angiography in newborn foals. Am. J. vet. Res. 36, 1021-1023.

Warren, R. G., Webb, A. I., Kosch, P. C., und Coons, L. L. (1984): Evaluation of transcutaneous oxygen monitoring in five anaesthetised pony foals. Equine vet. J. 16, 358-361.

Webb, A. I., Coons, T. J., Koterba, A. M., und Kosch, P. C. (1984 a): Developments in management of the newborn foal in respiratory distress: Treatment. Equine vet. J. 16, 319-323.

Webb, A. I., Daniel, R. T., Miller, H. S., und Kosch, P. C. (1984 b): Preliminary studies of the measurement of conjunctival oxygen tension in the foal. Am. J. vet. Res. (In press).

West, J. B. (1982): Pulmonary Pathophysiology: the Essentials, 2nd edn. Williams \& Wilkons, London. 180-186. 